\title{
MU head echo observations of the 2010 Geminids: radiant, orbit, and meteor flux observing biases
}

\author{
J. Kero ${ }^{1}$, C. Szasz ${ }^{1}$, and T. Nakamura ${ }^{2}$ \\ ${ }^{1}$ Swedish Institute of Space Physics (IRF), P.O. Box 812, 98128 Kiruna, Sweden \\ ${ }^{2}$ National Institute of Polar Research (NIPR), 10-3 Midoricho, Tachikawa, 190-8518 Tokyo, Japan \\ Correspondence to: J. Kero (kero@irf.se) \\ Received: 31 July 2012 - Revised: 23 January 2013 - Accepted: 2 February 2013 - Published: 6 March 2013
}

\begin{abstract}
We report Geminid meteor head echo observations with the high-power large-aperture (HPLA) Shigaraki middle and upper atmosphere (MU) radar in Japan $\left(34.85^{\circ} \mathrm{N}\right.$, $\left.136.10^{\circ} \mathrm{E}\right)$. The $\mathrm{MU}$ radar observation campaign was conducted from 13 December 2010, 08:00 UTC to 15 December, 20:00 UTC and resulted in $48 \mathrm{~h}$ of radar data. A total of $\sim 270$ Geminids were observed among $\sim 8800$ meteor head echoes with precisely determined orbits. The Geminid head echo activity is consistent with an earlier peak than the visual Geminid activity determined by the International Meteor Organization (IMO). The observed flux of Geminids is a factor of $\sim 3$ lower than the previously reported flux of the 2009 Orionids measured with an identical MU radar setup. We use the observed flux ratio to discuss the relation between the head echo mass-velocity selection effect, the mass distribution indices of meteor showers and the mass threshold of the MU radar.
\end{abstract}

Keywords. Atmospheric composition and structure (Middle atmosphere - composition and chemistry) - Interplanetary physics (Interplanetary dust) - Ionosphere (Ion chemistry and composition)

\section{Introduction}

Each year in mid-December, the Earth passes through a stream of dust giving rise to the Geminid meteor shower. The Geminid activity is very stable and has been observed every year in mid-December since 1862 (Jones, 1978). The discovery of this shower is surprisingly recent, given that the age of the Geminid meteoroid stream has been estimated to be of the order of thousands of years, according to several independent investigations and modelling approaches summarized by Ryabova (1999).

The widely recognized parent body of the Geminids is the asteroid (3200) Phaethon. Despite observational searches (e.g. Hsieh and Jewitt, 2005; Wiegert et al., 2008), no evidence of current mass loss from Phaethon was reported before an unexpected brightening by a factor of two that started $20.2 \pm 0.2$ UTC, June 2009 (Jewitt and Li, 2010). The brightening was interpreted as an impulsive release of dust particles with a combined mass of $\sim 2.5 \times 10^{8} \mathrm{~kg}$, or approximately $10^{-4}$ of the total Geminid stream mass.

Jewitt and Li (2010) suggest that Phaethon is a rock comet in which dust is produced by thermal fracture at the high surface temperatures $(\sim 1000 \mathrm{~K})$ experienced near perihelion $(q=0.14 \mathrm{AU})$. Particles smaller than $\sim 1 \mathrm{~mm}$ in radius cannot be held by Phaethon against radiation pressure this close to the Sun and are removed from the parent body irrespective of ejection velocity. Modelling work by Ryabova (2012) shows that the observed dust release in 2009 may give rise to a minor outburst exceeding the usual level of Geminid activity in 2017 at solar longitude $\lambda=262.5^{\circ}$.

Phaethon's spectral properties in the visible and nearinfrared are different from the properties of the few comet nuclei properly observed at these wavelengths and more similar to asteroid spectra (Licandro et al., 2007). Nevertheless, the observed structure of the regular Geminid stream agrees with the cometary scenario of its origin (Ryabova, 2001, 2007).

Specular meteor radar and visual observations of the Geminids contain abundant observational evidence of decreasing meteor magnitude (increasing meteoroid mass) as the shower progresses. The first evidence of a Geminid magnitude dispersion was provided by Plavcová (1962), using the Ondřejov radar $\left(49.55^{\circ} \mathrm{N}, 14.47^{\circ} \mathrm{E}\right)$. Subsequent 
investigations have been detailed, e.g. by Jones (1978); McIntosh and Simek (1980); Jones and Morton (1982); Simek and McIntosh (1989); Uchiyama (2010).

The reason for the Geminid magnitude dispersal is the mass-dependent radiative dispersal of meteoroid orbits, mainly due to the Poynting-Robertson (P-R) effect (e.g. Wyatt and Whipple, 1950; Briggs, 1962; Ryabova, 1999, and references therein), possibly with an equally large contribution from the Yarkovsky-Radzievskii effect (Olsson-Steel, 1987).

Brown et al. (2008, 2010); Blaauw et al. (2011) present results from the long-term observation programme conducted with the Canadian Meteor Orbit Radar (CMOR). CMOR has provided specular trail echoes in single-station operation since 1999 and multi-station orbit data since January 2002. Several million meteoroid orbits have been detected, and the radar data are continuously evaluated by several other methods including high-resolution optical measurements. Brown et al. (2010) found that the Geminid stream is broader and longer-lived at small radar particle sizes than had previously been appreciated (Sekanina, 1970). It extends from solar longitude $225^{\circ}$ to $282^{\circ}$; or roughly from 7 November to 2 January each year. Blaauw et al. (2011) investigated meteor shower mass distribution indices and found for the Geminids an index of 1.65 at peak shower activity.

Of the five major showers investigated by Blaauw et al. (2011), the Quadrantids and Geminids consistently had the lowest mass distribution indices, whereas the South Delta Aquariids, Eta Aquariids and Orionids had slightly higher ones. The mass index $s$ is related to the cumulative number $N_{\mathrm{c}}$ of meteors with mass $m$ or larger according to

$N_{\mathrm{c}} \propto m^{-(s-1)}$.

A mass index of 2 would imply that there is approximately the same mass in each size bin, while $s<2$ indicates that there is more mass in larger particles.

In this paper we present Geminid meteor head echo observations using the Shigaraki middle and upper atmosphere (MU) radar in Japan $\left(34.85^{\circ} \mathrm{N}, 136.10^{\circ} \mathrm{E}\right)$. The observations were conducted in support of the ECOMA (Existence and Charge state Of Meteoric dust particles in the Middle Atmosphere) sounding rocket campaign (Rapp and Robertson, 2009; Rapp et al., 2012) at the Andoya Rocket Range in Norway $\left(69.29^{\circ} \mathrm{N}, 16.02^{\circ} \mathrm{E}\right)$. The aim of ECOMA is to quantify the effect of the Geminids on the properties of meteoric smoke particles.

Section 2 gives a brief overview of meteor head echo observations. The MU radar was operated from 13 December 2010, 08:00 UTC to 15 December, 20:00 UTC in the meteor head echo mode outlined in Sect. 3.

Section 4 describes the MU radar Geminid observations. Section 4.1 focuses on the velocity and initial altitude, while Sect. 4.2 summarizes the activity and estimated flux. Section 4.3 expands upon the effects of observing biases and mass indices on the observed rate by comparing the Geminid flux with the previously reported flux of the 2009 Orionids (Kero et al., 2011).

\section{Meteor head echo radar observations}

A meteor head echo is caused by radio waves scattered from the dense region of plasma surrounding and co-moving with a meteoroid during atmospheric flight. The signal Doppler shift and/or range rate of the target can therefore be used to determine meteoroid velocity. The first head echoes were particularized by Hey et al. (1947) from observations using a $150 \mathrm{~kW}$ VHF radar system, conducted 7-11 October 1946, covering the anticipated 1946 Draconid meteor outburst.

Since the 1990s, head echo observations have been conducted utilizing most high-power large-aperture (HPLA) radar facilities around the world (e.g. Pellinen-Wannberg and Wannberg, 1994; Mathews et al., 1997; Close et al., 2000; Sato et al., 2000; Chau and Woodman, 2004; Mathews et al., 2008; Malhotra and Mathews, 2011). However, only a few observations of shower meteors have hitherto been published (e.g. Chau and Galindo, 2008; Kero et al., 2011, 2012a).

\section{MU radar experimental setup}

The $46.5 \mathrm{MHz} \mathrm{MU}$ radar has a nominal transmitter peak power of $1 \mathrm{MW}$ and comprises a circular, phased-array antenna with a diameter of $103 \mathrm{~m}$. The antenna field consists of 475 crossed Yagi antennas with one transmitter/receiver module each (Fukao et al., 1985), and has a one-way $-3 \mathrm{~dB}$ full beam width of $3.6^{\circ}$. The beam width is similar to that of The Middle Atmosphere Alomar Radar System (MAARSY; Latteck et al., 2010) but is wider than for most other HPLA radar systems. This gives a comparatively large observing volume and, consequently, a larger possibility of observing longer meteor trajectories.

The 25-channel digital receiver system of the MU radar is described by Hassenpflug et al. (2008). In our meteor head echo observations, the output from 25 subgroups of 19 Yagi antennas were each stored as the data of separate digital channels. The receiver system was upgraded from a 4channel setup in 2004. Meteor head echo observations prior to the upgrade have been reported by Sato et al. (2000) and Nishimura et al. (2001).

We have developed improved analysis algorithms (Kero et al., 2012c) and collected an extensive set of data (>500 h) between June 2009 and December 2010. Kero et al. (2012b) give an initial overview of the whole data set including radiant density and orbital properties of sporadic meteors. Kero et al. (2011, 2012a) describe investigations of head echoes associated with comet 1P/Halley dust of the 2009 Orionid meteor shower and comet 21P/Giacobini-Zinner dust of the October 2011 Draconid outburst.

During the Geminid observation, the MU radar was configured to run with settings identical to those in the 


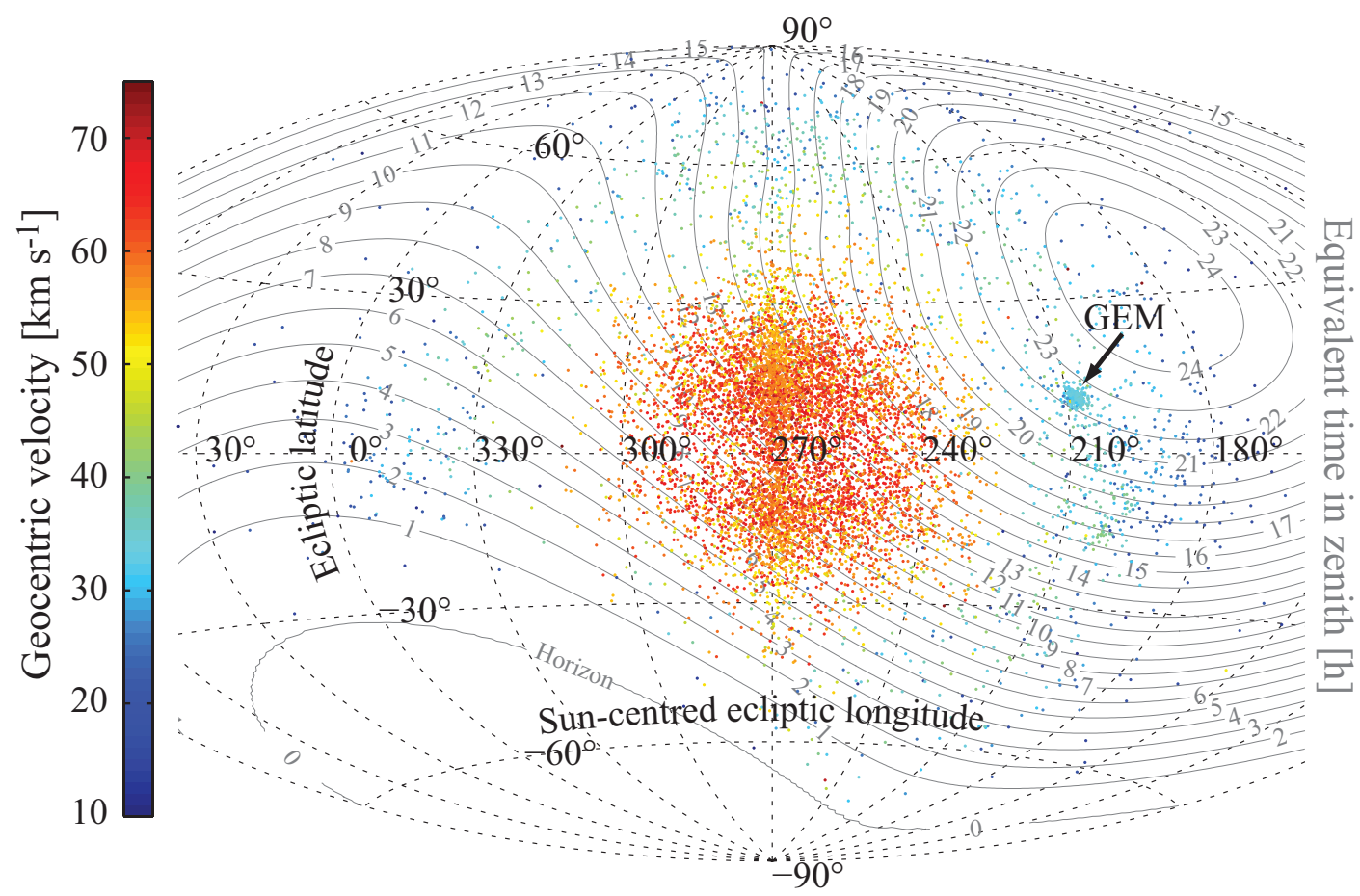

Fig. 1. The radiant distribution of $\sim 8800$ meteors plotted in ecliptic latitude and Sun-centred ecliptic longitude. Geocentric velocity is colour-coded. The Geminid (GEM) shower radiant is marked by an arrow. Solid lines map the equivalent time in zenith for different regions of the celestial sphere. One hour in the plot corresponds to one hour in zenith. The region of the celestial sphere below the line marked "Horizon" was always below the Shigaraki local horizon.

previously reported measurements. The MU radar beam was pointed in the direction of zenith. Head echoes were detected in the height range of $73-127 \mathrm{~km}$, limited by the experimental settings being adapted to the maximum data rate of $20 \mathrm{~GB} \mathrm{~h}^{-1}$. We transmitted 13-bit Barker-coded pulses with a total pulse length of $156 \mu$ s and an interpulse period (IPP) of $3.12 \mathrm{~ms}$ to use the $5 \%$ duty cycle.

The received data were stored as 85 range values from each transmitted pulse, sampled at $6 \mu$ s intervals. The sampling corresponds to a range resolution of about $900 \mathrm{~m}$. Nevertheless, using a novel range interpolation technique (Kero et al., 2012c), the ranges of meteor targets are determined with a precision of the order of $10 \mathrm{~m}$, or to within about onehundredth of a range gate.

Meteor targets are searched for within $15^{\circ}$ from the nominal beam direction (zenith). The 25-channel setup enables unambiguous directionality and the possibility of distinguishing head echo targets in the main lobe region from head echo targets in the side lobes.

There are no unintentional gaps in the data set. The radar is, however, stopped and restarted every full hour to reboot the system and avoid memory buffer overflow, an occasional consequence of the data rate being close to the system limit. Because of this procedure we have no data for the first 12 min of every full hour.

\section{Geminid observations}

Figure 1 gives an overview of the MU 2010 Geminid data set. The radiant distribution is displayed in Sun-centred ecliptic coordinates. The Sun is in this coordinate system always located at $0^{\circ}$ Sun-centred ecliptic longitude, regardless of time of year. The direction of the Earth's apex is located at $270^{\circ}$ Sun-centred ecliptic longitude.

The MU sporadic meteor distribution is dominated by the north and south apex apparent source regions, further discussed in Kero et al. (2012b). Solid lines mark the equivalent time in zenith for different regions of the celestial sphere, estimated according to the procedure described in Kero et al. (2012b). One hour in the plot corresponds to one hour in zenith. The line marked "Horizon" represents the Shigaraki local horizon.

A compact radiant enhancement appears at the expected radiant region of the Geminids (GEM) and consists of about 270 meteors. Figure 2 shows the radiant density near the enhancement in equatorial coordinates, reduced to solar longitude $261.0^{\circ}$ by taking into account the radiant drift reported by Brown et al. (2010). The mean Geminid radiant is located at right ascension $\alpha=112.8^{\circ} \pm 0.5^{\circ}$ and declination $\delta=32.6^{\circ} \pm 0.3^{\circ}$. The errors represent the standard deviation of the mean, calculated using the individual radiants and their estimated uncertainty margins. 


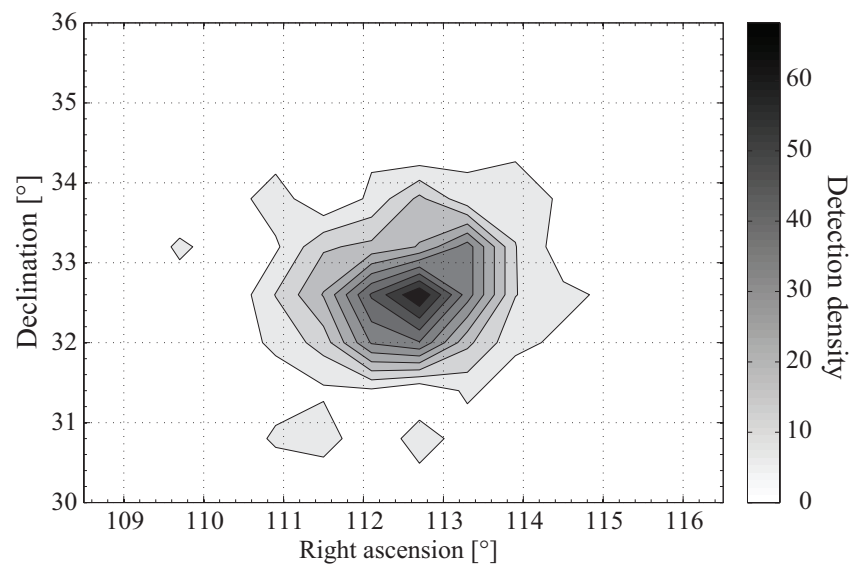

Fig. 2. Geminid radiant density plot in equatorial coordinates, reduced to solar longitude $261.0^{\circ}$.

The mean value of the uncertainty of individual radiants is $0.8^{\circ}$ in local horizontal coordinates (azimuthal and zenithal distance uncertainty). When these uncertainties are propagated through the conversion to equatorial coordinates $(\alpha, \delta)$, the mean radiant measurement error is $1^{\circ}$. This value is comparable to the full width at half maximum of the radiant density (Fig. 2). The measured width of the shower is, therefore, likely defined by the measurement errors.

The mean Geminid heliocentric orbit as measured with MU is summarized in Table 1, together with other reported radar orbit results and the orbit of Phaethon. Error margins of the MU orbit parameters are estimated according to the uncertainty calculations detailed in Appendix A of Kero et al. (2012a). The mean semi-major axis of the MU radar Geminids is $1.32 \pm 0.06 \mathrm{AU}$ and the mean period is $1.49 \pm 0.06 \mathrm{yr}$. These numbers are similar to the values derived in other studies, presented in Table 1 and further summarized by Jenniskens (2006).

\subsection{Geminid velocity and initial altitude}

Figure 3 is a scatter plot of meteor initial detection altitude versus geocentric velocity, corrected for Earth focusing (zenith attraction) and Earth rotation following Szasz et al. (2008) and Szasz (2008). The Geminids are plotted in a contrasting colour and compared to a linear least squares fit (black line). The fitted Geminid geocentric velocity is $34.9 \mathrm{~km} \mathrm{~s}^{-1}$ at an altitude of $109.5 \mathrm{~km}$ and decreases with $0.11 \mathrm{~km} \mathrm{~s}^{-1}$ for each kilometre of lower initial height. The value of the observed velocity (before correction for Earth encounter) at $109.5 \mathrm{~km}$ was $36.6 \mathrm{~km} \mathrm{~s}^{-1}$.

The trend means that some particles have likely experienced deceleration (and ablation) before detection. If so, their initial detection altitude is not equal to the true beginning height of the meteor. In the orbit calculation, we have used the velocity found for the highest altitude Geminids as representative of the atmospheric entry velocity of the Geminid

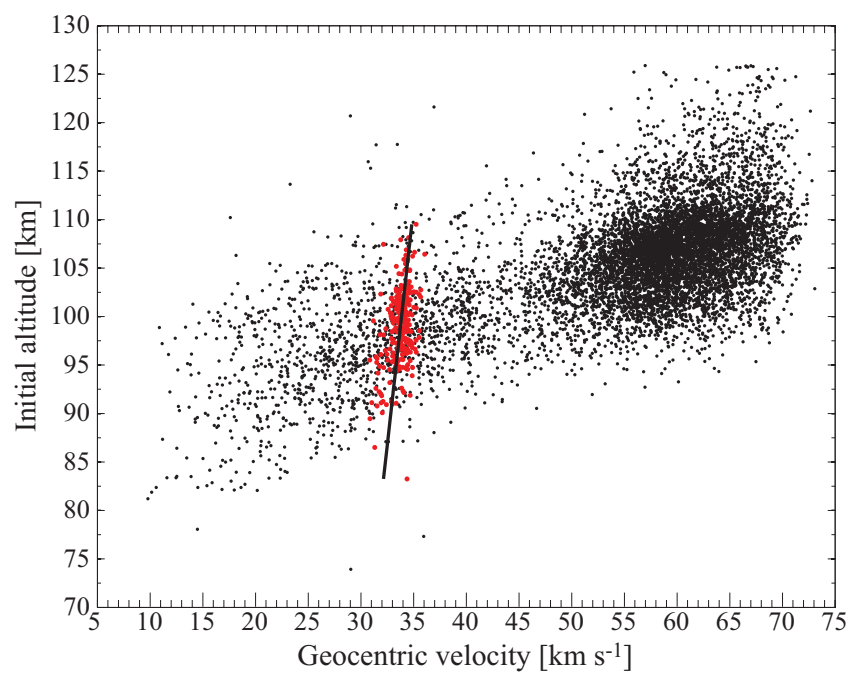

Fig. 3. Scatter diagram of initial detection altitude versus geocentric velocity of sporadic meteors (black) and the Geminids (red). The solid line is a linear least-squares fit to the Geminid geocentric velocity, giving $34.9 \mathrm{~km} \mathrm{~s}^{-1}$ at $109.5 \mathrm{~km}$ altitude.

shower. A backward integration of the observed velocity profiles to the top of the atmosphere using an ablation model (e.g. Kero, 2008; Stober et al., 2011) is an alternative way to find accurate entry velocities.

The Geminid initial velocity and altitude trend is very similar to the trend found for the 2011 October Draconid meteors (Kero et al., 2012a). On the other hand, while the initial detection altitudes of the Geminids are comparable to the altitudes of sporadic meteors of the same velocity, the initial altitudes of the MU radar October 2011 Draconids were systematically higher.

The difference in initial altitudes is likely a manifestation of the different material properties of Geminid meteoroids originating from 3200 Phaethon and fresh dust from comet 21P/Giacobini-Zinner giving rise to the Draconid shower. Geminid meteoroids do not only come from a very different parent body, but have also frequently been exposed to intense solar radiation near perihelion at $0.14 \mathrm{AU}$, a possible consequence of which may be loss of volatiles (Wetherill, 1986; Jenniskens, 2006). Geminid fireballs have been found to be more fragile than fireballs of asteroidal meteoroids of the same size, but more cohesive than other cometary shower ones (Jenniskens, 2006, and references therein).

When comparing showers that occur during different times of the year, it is important to remember that the background neutral atmospheric density varies according to a distinct seasonal pattern. Atmospheric waves also give rise to a density modulation. As shown by e.g. Younger et al. (2009); Stober et al. (2012), the density can be as variable as 10-20\%. 


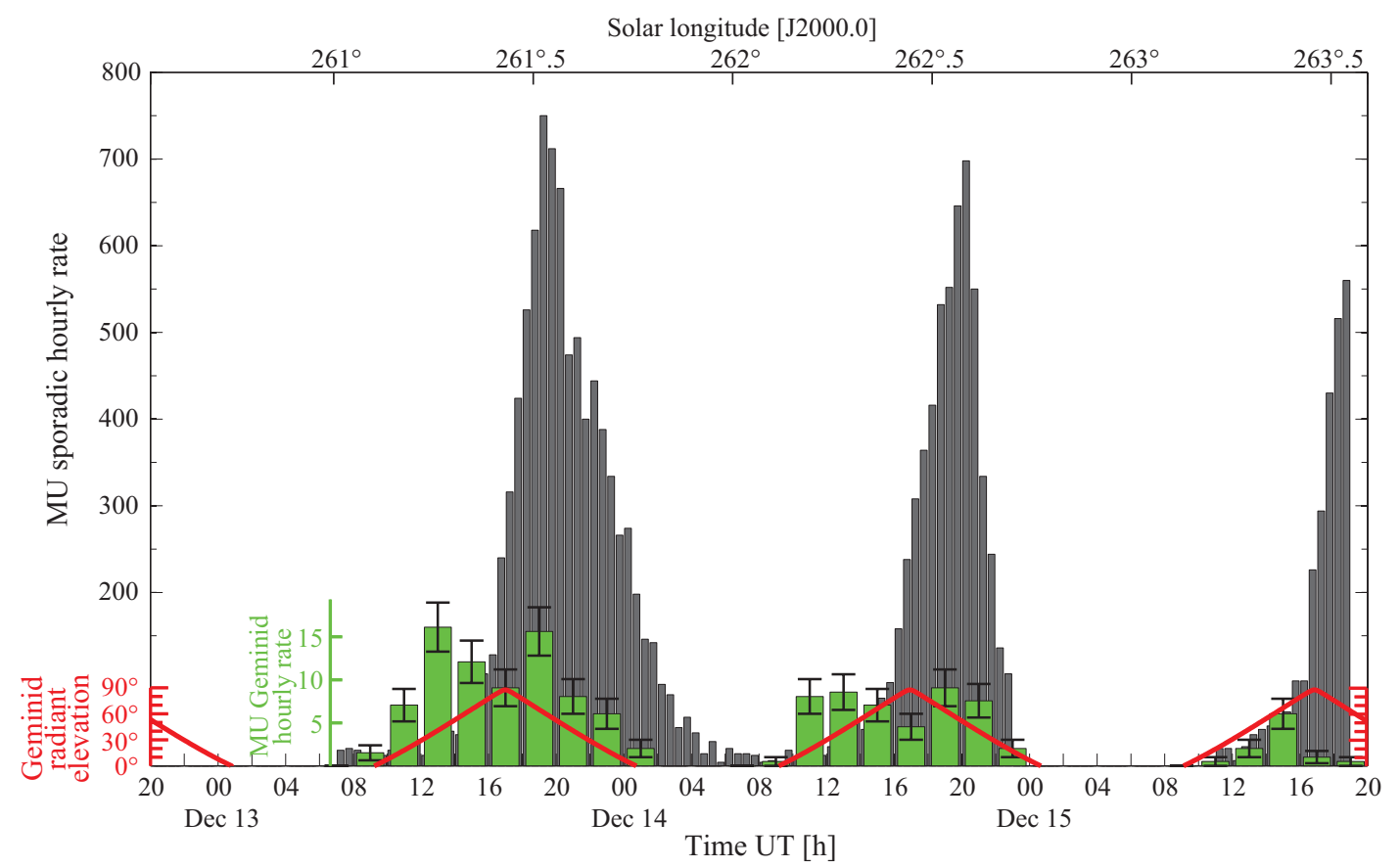

Fig. 4. Histogram of the hourly detection rate of MU meteor head echoes with precisely determined orbits detected during the Geminid campaign. The sporadic hourly meteor rate (grey) is given for each half-hour interval. Due to the relatively low number of MU radar Geminids, the Geminid hourly rate (green) is presented for every two-hour interval and error bars (black) correspond to the estimated Poisson uncertainty. The elevation of the Geminid radiant (red) peaked around 17:00 UT.

\subsection{The Geminid activity and estimated flux}

Figure 4 displays the hourly rate of MU meteor head echoes with precisely determined orbits detected during the Geminid campaign. The sporadic hourly meteor rate (grey) is given for each half-hour interval. Because of the relatively low number of MU radar Geminids, the Geminid hourly rate (green) is presented for every two-hour interval. Error bars (black) correspond to the estimated Poisson uncertainty. The elevation of the Geminid radiant above the local horizon is plotted as a curve (red).

The MU Geminid count rate drops near both shower radiant culminations (at $\sim 17: 00 \mathrm{UT}$ ) covered during the observation. We find that the strengths of the minima depend on how the data are binned. These count rate minima are therefore partly explained by statistical fluctuations due to the relatively low number of Geminids (about 10 per hour). Another contributory factor is the variation of the Geminid activity. In Fig. 5, we have compensated the count rate for radiant elevation (explained below) and plotted the scaled rate together with the visual zenithal hourly rate (ZHR) reported by the International Meteor Organization (IMO). The latter was calculated by G. Barentsen from 6801 visually observed Geminids collected by the IMO. Both of the dips in the MU scaled rate near radiant culmination coincide with dips in the visual activity. The MU radar activity dips are deeper, which

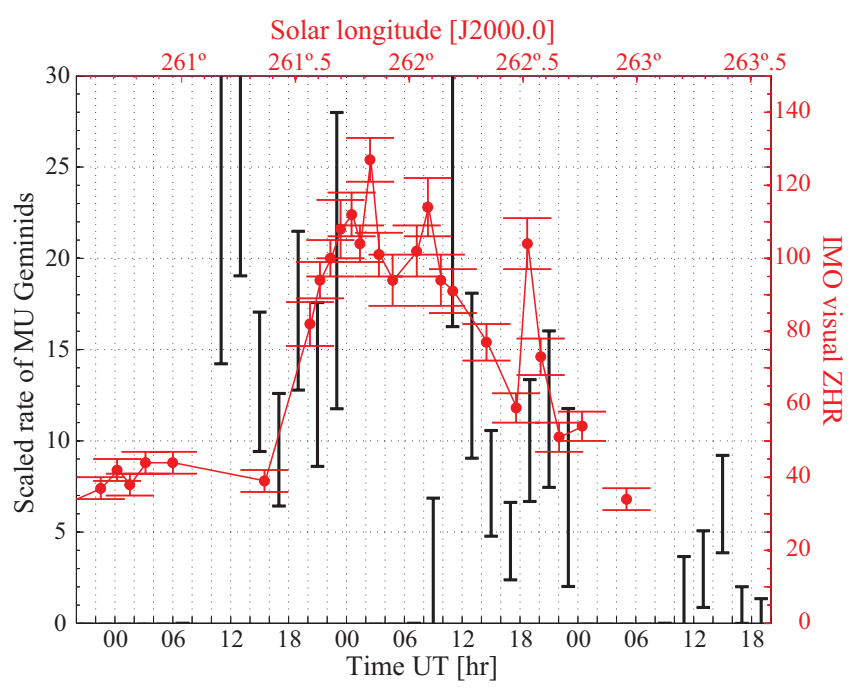

Fig. 5. MU radar scaled rate of Geminids (black) and the visual ZHR (red) calculated by G. Barentsen from 6801 visually observed Geminids collected by the International Meteor Organization (IMO). The vertical bars represent Poisson-distributed statistical errors of the meteor counts.

may indicate a greater decrease of low-mass Geminids than of visual Geminids.

An additional effect may arise from the effective collection area of the vertically aligned MU radar beam pattern. 
Table 1. Mean heliocentric orbit (J2000.0) of MU, CMOR, HRMP and the Kharkov radar Geminids, as well as the orbit of parent body 3200 Phaethon (1983 TB): semi-major axis $(a)$, eccentricity $(e)$, perihelion distance $(q)$, longitude of the ascending node $(\Omega)$, inclination $(i)$, argument of perihelion $(\omega)$, period $(P)$, and number of detected orbits $\left(N_{\text {orb }}\right)$. For MU, $\Omega$ denotes the range of the Geminid ascending node (in solar longitude) covered during the observation. For CMOR, HRMP and Kharkov, the value of $\Omega$ refers to ascending node at maximum activity.

\begin{tabular}{|c|c|c|c|c|c|c|c|c|}
\hline & $a[\mathrm{AU}]$ & $e$ & $q[\mathrm{AU}]$ & $\Omega\left[^{\circ}\right]$ & $i\left[{ }^{\circ}\right]$ & $\omega\left[^{\circ}\right]$ & $P$ [yr] & $N_{\text {orb }}$ \\
\hline $\mathrm{CMOR}^{\mathrm{a}}$ & 1.35 & 0.898 & 0.1373 & 261.0 & 23.2 & 324.95 & 1.57 & 10381 \\
\hline Kharkov ${ }^{\mathrm{c}}$ & 1.31 & 0.89 & 0.14 & 260 & 24 & 326 & 1.5 & 401 \\
\hline Phaethon $^{\mathrm{d}}$ & 1.271 & 0.8901 & 0.1397 & 265.281 & 22.234 & 322.132 & 1.43 & \\
\hline
\end{tabular}

${ }^{\text {a }}$ Brown et al. (2010)

b Sekanina (1976)

c Kashcheyev and Lebedinets (1967)

d JPL Small-Body Database, Orbital Elements at Epoch 2456200.5 (30 Sep 2012)

For Orionids and sporadic meteors, Kero et al. (2011) found count rate (and, consequently, the effective collection area) to follow $\sin ^{\gamma}$ el, where the radiant altitude exponent is $\gamma \sim$ 1.5 , and "el" is the radiant elevation. For visual meteors, $\gamma$ is generally determined to have a value in the range of $1<\gamma<2$, depending on meteor shower, varying slightly between different investigations (Jenniskens, 1994, and references therein). Interestingly, $\gamma \sim 1.5$ found for MU 2009 Orionids agree well with the theoretical exponent found by Jenniskens (1994) for visual Orionids and the empirical value of $\gamma=1.47 \pm 0.11$ in the pioneering work of Zvolankova (1983).

In Fig. 5 we have normalized the 2010 Geminid count rate using $\gamma=1.0$. A larger value of $\gamma$ produces an activity that is strongly anti-correlated with the radiant elevation. This is more likely a sign of an erroneously chosen altitude exponent than a true variation of the activity.

According to the IMO data (Fig. 4), the visual Geminid activity peaked around 14 December, 03:00 UTC, or at a solar longitude of about $261.8^{\circ}$. The MU head echo Geminid activity indicates an earlier maximum activity. Since there are no data (the radiant was below the local horizon) during the visual peak, a peak simultaneous with the visual activity cannot be ruled out.

The MU scaled rate has an average value of about $15 \mathrm{~h}^{-1}$ during the first half of the observation. We have used this value to estimate the cumulative flux of Geminid meteors down to the Geminid mass threshold of the MU radar following the procedure described in Kero et al. (2011) for the flux estimation of the 2009 Orionids. To accomplish this we first binned the Geminid meteors according to their maximum RCS. As a second step, we calculated the cumulative flux as a function of RCS using the RCS dependent effective collection area presented in Fig. 7 of Kero et al. (2011).

The resulting cumulative Geminid flux is in Fig. 6 presented together with the 2009 Orionid flux, adapted from Fig. 14 of Kero et al. (2011). The 2010 Geminid cumulative

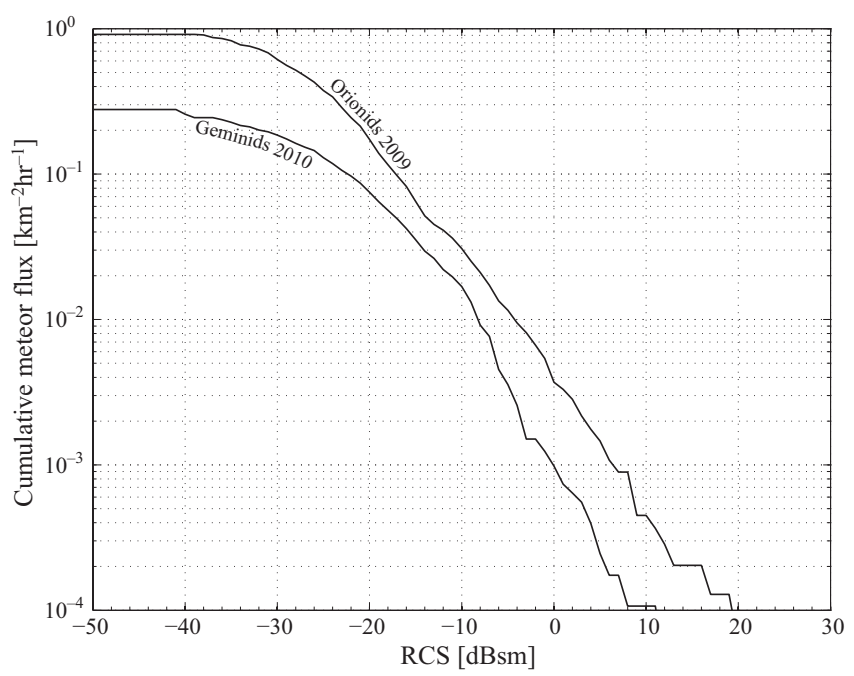

Fig. 6. Cumulative meteor flux versus RCS for the 2010 Geminids, and as a comparison the 2009 Orionids adapted from Kero et al. (2011).

flux is $\sim 0.3 \mathrm{~h}^{-1} \mathrm{~km}^{-2}$, or about a factor of three lower than the $\sim 1 \mathrm{~h}^{-1} \mathrm{~km}^{-2} 2009$ Orionid flux. The determined values of the fluxes strongly depend on a correct estimation of the effective collection area.

\subsection{The Geminid to Orionid flux ratio}

Meteor head echo observing biases, as the ionization efficiency, are not fully understood yet and introduce significant uncertainties in deriving meteoroid masses (CampbellBrown et al., 2012). The Geminid stream is the highest flux visual shower currently visible from Earth (Rendtel et al., 1995). The lower observed MU radar flux of Geminids than Orionids therefore requires a digression on the factors controlling the observability of head echoes. 

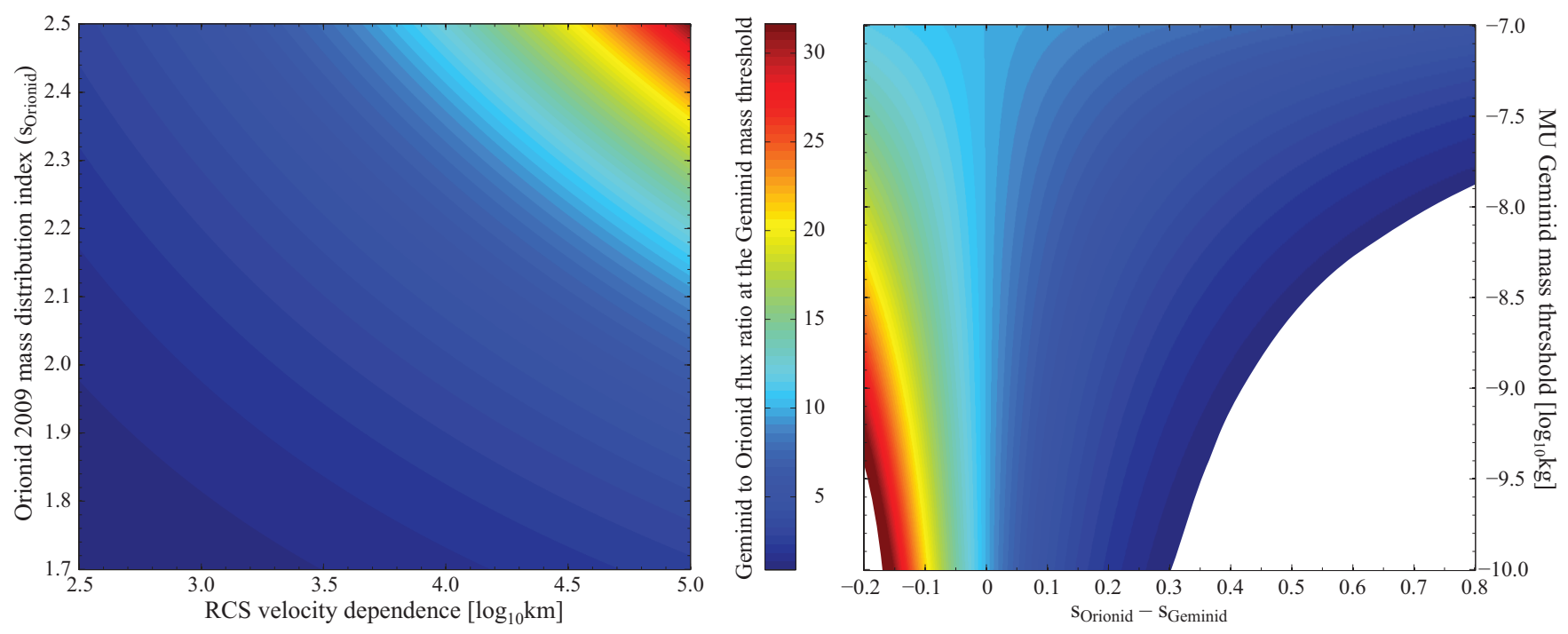

Fig. 7. Left panel: ratio of the Geminid to the Orionid cumulative fluxes, normalized to the Geminid mass threshold, when their observed ratio is 0.3. Right panel: ratio of the Geminid to the Orionid cumulative fluxes, normalized to the Geminid mass threshold, if their flux ratio is 10 at a limiting mass of $2 \times 10^{-7} \mathrm{~kg}$.

One such factor is a frequency dependent altitude cut-off. For specular meteor trail echoes, this is called the initial trail radius attenuation effect and was first described by Greenhow and Hall (1960), who compared the altitude distributions of three radar systems operating at different frequencies. If the initial train radius is large compared to the radar wavelength, strong attenuation arises owing to destructive interference from radio waves scattered at different radial depths of the train. It is particularly severe for high-speed meteors, as these ablate at high altitudes. There the atmospheric mean free path and thus also the initial train radii are large.

In the case of the Orionids, Jones (1983) estimated that $<3 \%$ of the total number was detected. Such a grave attenuation introduces large uncertainties when converting the observed number of detections to meteor influx.

Westman et al. (2004) compared meteor observations from the EISCAT VHF $(224 \mathrm{MHz})$ and UHF $(930 \mathrm{MHz})$ radars. They found that the detection altitude distributions are similar, except for the upper part of the UHF altitude distribution being cut several km below the upper part of the VHF distribution. The much lower MU radar operating frequency of $46.5 \mathrm{MHz}$, and its correspondingly longer wavelength, means that the effects of altitude cut-off are probably weaker. We have in the remainder of this paper assumed that the altitude cut-off effect can be neglected in the case of MU. We will address the MU altitude distribution and possible cut-off issues in a subsequent investigation.

Another controlling factor is the dependence of ionization efficiency on meteoroid velocity. Close et al. (2007) investigated mass-velocity selection effects on head echoes detected with the Advanced Research Projects Agency LongRange Tracking and Instrumentation Radar (ALTAIR). Their derived dependence of radar cross section (RCS) on electron line density $Q$ is

$\mathrm{RCS} \propto(-42.4 \pm 1.2) Q^{1.05 \pm 0.04}$

where $Q$ is found to depend on mass $(m)$, velocity $(v)$ and mean free path (mfp) according to

$Q \propto m^{1.08 \pm 0.08} v^{3.09 \pm 0.66} \mathrm{mfp}^{-0.11 \pm 0.18}$.

Close et al. (2007) used Eq. (2) and (3) to normalize the ALTAIR observations together with an estimated mass index $s=1.94$. These values translate to a velocity-over-mass ratio $(\xi)$ of 2.9 for the ionization efficiency.

Previous investigations of the ionization efficiency have yielded slightly higher values of $\xi$. Verniani and Hawkins (1964) found from comparisons of radar trail echo and photographic studies a value of $\xi=4$ and Bronshten (1983) reported $\xi=3.75$ based on laboratory measurements of chondritic atoms. The Jones (1997) model of ionization efficiency is limited to processes without significant secondary ionization or recombination and is therefore in principle valid only for faint radio meteors with $v \lesssim 35 \mathrm{~km} \mathrm{~s}^{-1}$. Jones estimates that the primary ionization varies with $\xi \simeq 3.4$.

Hunt (2007) has utilized ALTAIR data in an effort to derive an ionization coefficient $(\beta)$ that is more physically representative than that of the Jones model for highly energetic collisions. Hunt finds

$\beta=2 \times 10^{-9}(v-11) v^{4}$,

where the meteoroid velocity $v$ should be given in $\mathrm{km} \mathrm{s}^{-1}$. This formulation corresponds to $\xi \simeq 5.3$ in our velocity range. 
Szasz et al. (2008) estimated meteoroid masses of tristatic EISCAT UHF meteors dynamically by comparing the measured velocity and deceleration to the output of an ablation model. The procedures are further described by Kero et al. (2008); Kero (2008). The lowest meteoroid mass producing a detectable meteor as a function of velocity was used as a crude estimate of the ionization efficiency of the observed particles. A least-squares fit of a linear function to the EISCAT UHF mass-velocity distribution corresponded to $\xi \simeq 4.1 \pm 0.4$. The regression fit had a curvilinear residual distribution, which indicated that $\xi$ actually may be velocity dependent with a larger value of $\xi$ in the low-velocity end of the observations than in the high-velocity end.

The left panel of Fig. 7 shows the ratio of the Geminid to the Orionid cumulative fluxes, normalized to the Geminid mass threshold, when their observed ratio is 0.3 . The horizontal axis displays the exponent of the RCS velocity dependence (assuming that the RCS mass dependence exponent is unity and mean-free-path exponent is zero). The vertical axis visualizes the 2009 Orionid mass distribution index, $s_{\text {Orionid, }}$ while the colour bar shows the estimated ratio. Within the range of velocity dependence and Orionid mass distribution index displayed in Fig. 7, the ratio of the cumulative fluxes at the Geminid mass threshold varies between $\sim 1-30$.

Jones (1983) calculated that a peak visual Orionid ZHR of $22 \mathrm{~h}^{-1}$ corresponds to a flux of $\sim 0.004 \mathrm{~h}^{-1} \mathrm{~km}^{-2}$ Orionids brighter than magnitude +4.25 when taking into account the most appropriate detection probability function of the data set (Stohl and Porubcan, 1981; Kresáková, 1966). We use this value as a canonical flux of +4.25 magnitude Orionids.

Brown et al. (1998) investigated the radar flux of Geminids and found a peak flux of $0.19 \pm 0.2 \mathrm{~h}^{-1} \mathrm{~km}^{-2}$ for radio magnitude +7.7 . Except for the very brief peak, the flux of magnitude class +7.7 was $0.12 \pm 0.2 \mathrm{~h}^{-1} \mathrm{~km}^{-2}$ for solar longitude $260.5-261.5^{\circ}$. Furthermore, Brown et al. (1998) report that the flux of visual Geminids down to limiting visual magnitude +6.5 had a peak value of $\sim 0.04 \mathrm{~h}^{-1} \mathrm{~km}^{-2}$.

Verniani (1973) found that magnitude $(M)$ depends on mass $(m)$ and velocity $(v)$ according to

$M=63.17-10 \lg (v)-2.5 \lg (m)$

in cgs units (mass given in gram and velocity in $\mathrm{cm} \mathrm{s}^{-1}$ ). The visual magnitude of a $2 \times 10^{-7} \mathrm{~kg}$ meteoroid is +4.3 at the Orionid velocity and +6.8 at the Geminid velocity. These values are close to the above-reported limiting visual magnitudes.

Using the ratio of the estimated visual fluxes, we therefore assume that the flux ratio of $2 \times 10^{-7} \mathrm{~kg}$ meteoroids in the Geminid and Orionid showers has a value of $\sim 10$. The right panel of Fig. 7 displays what the flux ratio (colour bar) under the discussed assumptions would be at the MU Geminid mass threshold as a function of the showers' difference in mass distribution indices, $s_{\text {Orionid }}-s_{\text {Geminid }}$ (horizontal axis), and the MU Geminid mass threshold (vertical axis).
Figure 7 can be seen as a lookup table to study the effects of velocity dependence and mass distribution indices on mass threshold estimations. As an example, if the RCS velocity dependence were 4 and $s_{\text {Orionid }}=1.9$, looking at the colour scale in the left panel gives a flux ratio at the MU Geminid mass threshold of $\sim 3$. Furthermore, if assuming e.g. $s_{\text {Geminid }}=1.7$, the right panel shows that for the given ratio of $\sim 3$ (looking at the same colour as was found in the left panel), the MU Geminid mass threshold has a value of $\sim 10^{-9} \mathrm{~kg}$. The so-determined mass threshold is virtually independent of whether the MU radar collection area reported by Kero et al. (2011) was correctly determined or not, as only the ratio of Geminid and Orionid fluxes is used, whereas the absolute values of the fluxes are not. However, the mass threshold is very sensitive to small changes in the assumed shower mass distribution indices and the velocity dependence.

It is not trivial to convert observed visual, photographic or radar magnitude distributions to mass distributions, not even for such a well-studied meteor shower as the Geminids. Excellent examples of issues that arise when converting magnitude to mass are given in the work by Ceplecha (1957), who investigated photographic Geminids.

\section{Conclusions}

A total number of $\sim 270$ Geminids, among $\sim 8800$ meteor head echoes with precisely determined orbits, were observed with the MU radar during a campaign conducted from $13 \mathrm{De}-$ cember 2010, 08:00 UTC to 15 December, 20:00 UTC. The fitted MU radar Geminid geocentric velocity is $34.9 \mathrm{~km} \mathrm{~s}^{-1}$ at an altitude of $109.5 \mathrm{~km}$ and decreases with $0.11 \mathrm{~km} \mathrm{~s}^{-1}$ for each kilometre of lower initial height. The observed velocity at $109.5 \mathrm{~km}$ is $36.6 \mathrm{~km} \mathrm{~s}^{-1}$ before compensating for Earth encounter. The mean radiant, reduced to solar longitude $261.0^{\circ}$ by taking into account the radiant drift reported by Brown et al. (2010), is located at right ascension $\alpha=112.8^{\circ} \pm 0.5^{\circ}$ and declination $\delta=32.6^{\circ} \pm 0.3^{\circ}$.

The visual Geminid activity reported to the IMO peaked around 14 December 2010, 03:00 UTC, at a solar longitude of about $261.8^{\circ}$. The MU head echo Geminid activity is consistent with an earlier-occurring maximum. The mean semi-major axis and period of the head echo meteoroids are $1.32 \pm 0.06 \mathrm{AU}$ and $1.49 \pm 0.06 \mathrm{yr}$, respectively. These values agree with observations using other methods and an estimated mass range from about 0.5 down to $10^{-9} \mathrm{~kg}$ (Jenniskens, 2006).

The observed MU Geminid cumulative flux is $\sim 0.3 \mathrm{~h}^{-1} \mathrm{~km}^{-2}$. This value is about a factor of three lower than the previously reported flux of the 2009 Orionids, $\sim 1 \mathrm{~h}^{-1} \mathrm{~km}^{-2}$, measured with an identical MU radar setup (Kero et al., 2011).

The determined flux values depend strongly on a correct estimation of the MU effective collection area, while their 
ratio does not. We therefore used the observed flux ratio to discuss the relation between the head echo mass-velocity selection effect, the mass distribution indices of meteor showers, and the mass threshold of the MU radar. We found that the mass threshold is very sensitive to small changes in the shower mass distribution index assumptions as well as in the assumed dependence of ionization efficiency on meteoroid velocity.

Acknowledgements. This study is supported by JSPS grant-in-aid 24340121. The MU radar belongs to and is operated by the Research Institute of Sustainable Humanosphere, Kyoto University, Uji, Kyoto, Japan. The authors would like to thank Gunter Stober and an anonymous reviewer for valuable comments and suggestions that improved the manuscript.

Guest Editor M. Rapp thanks G. Stober and one anonymous referee for their help in evaluating this paper.

\section{References}

Blaauw, R. C., Campbell-Brown, M. D., and Weryk, R. J.: A meteoroid stream survey using the Canadian Meteor Orbit Radar - III. Mass distribution indices of six major meteor showers, MNRAS, 414, 3322-3329, doi:10.1111/j.1365-2966.2011.18633.x, 2011.

Briggs, R. E.: Symposium: Astrometry I: The steady-state space distribution of meteoric particles under the operation of the Poynting-Robertson Effect, ApJ, 67, 710, doi:10.1086/108799, 1962.

Bronshten, V. A.: Physics of meteoric phenomena, D. Reidel Publishing Company, 1983.

Brown, P., Hocking, W. K., Jones, J., and Rendtel, J.: Observations of the Geminids and Quadrantids using a stratospheretroposphere radar, MNRAS, 295, 847-859, doi:10.1046/j.13658711.1998.01279.x, 1998.

Brown, P., Weryk, R. J., Wong, D. K., and Jones, J.: A meteoroid stream survey using the Canadian Meteor Orbit Radar. I. Methodology and radiant catalogue, Icarus, 195, 317-339, doi:10.1016/j.icarus.2007.12.002, 2008.

Brown, P., Wong, D. K., Weryk, R. J., and Wiegert, P.: A meteoroid stream survey using the Canadian Meteor Orbit Radar. II: Identification of minor showers using a 3D wavelet transform, Icarus, 207, 66-81, doi:10.1016/j.icarus.2009.11.015, 2010.

Campbell-Brown, M. D., Kero, J., Szasz, C., Pellinen-Wannberg, A., and Weryk, R. J.: Photometric and ionization masses of meteors with simultaneous EISCAT UHF radar and intensified video observations, J. Geophys. Res. SP, 117, A09323, doi:10.1029/2012JA017800, 2012.

Ceplecha, Z.: Photographic Geminids 1955, Bulletin of the Astronomical Institutes of Czechoslovakia, 8, 51, 1957.

Chau, J. L. and Galindo, F.: First definitive observations of meteor shower particles using a high-power large-aperture radar, Icarus, 194, 23-29, doi:10.1016/j.icarus.2007.09.021, 2008.

Chau, J. L. and Woodman, R. F.: Observations of meteor-head echoes using the Jicamarca $50 \mathrm{MHz}$ radar in interferometer mode, Atmos. Chem. Phys., 4, 511-521, doi:10.5194/acp-4-5112004, 2004.
Close, S., Hunt, S. M., Minardi, M. J., and McKeen, F. M.: Analysis of Perseid meteor head echo data collected using the Advanced Research Projects Agency Long-Range Tracking and Instrumentation Radar (ALTAIR), Radio Sci., 35, 1233-1240, doi:10.1029/1999RS002277, 2000.

Close, S., Brown, P., Campbell-Brown, M., Oppenheim, M., and Colestock, P.: Meteor head echo radar data: Mass velocity selection effects, Icarus, 186, 547-556, doi:10.1016/j.icarus.2006.09.007, 2007.

Fukao, S., Sato, T., Tsuda, T., Kato, S., Wakasugi, K., and Makihira, T.: The MU radar with active phased array system. I - Antenna and power amplifiers. II - In-house equipment, Radio Sci., 20, 1155-1176, doi:10.1029/RS020i006p01155, 1985.

Greenhow, J. S. and Hall, J. E.: The importance of initial trail radius on the apparent height and number distributions of meteor echoes, MNRAS, 121, 183-196, 1960.

Hassenpflug, G., Yamamoto, M., Luce, H., and Fukao, S.: Description and demonstration of the new Middle and Upper atmosphere Radar imaging system: 1-D, 2-D, and 3-D imaging of troposphere and stratosphere, Radio Sci., 43, RS2013, doi:10.1029/2006RS003603, 2008.

Hey, J. S., Parsons, S. J., and Stewart, G. S.: Radar observations of the Giacobinids meteor shower, 1946, MNRAS, 107, 176-183, 1947.

Hsieh, H. H. and Jewitt, D.: Search for Activity in 3200 Phaethon, ApJ, 624, 1093-1096, doi:10.1086/429250, 2005.

Hunt, S.: Determination of the meteoroid ionization coefficient using high power large aperture radar, Ph.D. thesis, Boston University, United States - Massachusetts, 2007.

Jenniskens, P.: Meteor stream activity I. The annual streams, A\&A, 287, 990-1013, 1994.

Jenniskens, P.: Meteor Showers and their Parent Comets, Cambridge University Press, New York, USA, 2006.

Jewitt, D. and Li, J.: Activity in Geminid Parent (3200) Phaethon, AJ, 140, 1519-1527, doi:10.1088/0004-6256/140/5/1519, 2010.

Jones, J.: On the period of the Geminid meteor stream, MNRAS, 183, 539-546, 1978.

Jones, J.: Radar observations of the Orionid meteor shower, MNRAS, 204, 765-776, 1983.

Jones, J. and Morton, J. D.: High-resolution radar studies of the Geminid meteor shower, MNRAS, 200, 281-291, 1982.

Jones, W.: Theoretical and observational determinations of the ionization coefficient of meteors, MNRAS, 288, 995-1003, 1997.

Kashcheyev, B. L. and Lebedinets, V. N.: Radar studies of meteors, Smithsonian Contributions to Astrophysics, 11, 183-199, 1967.

Kero, J.: High-resolution meteor exploration with tristatic radar methods, Ph.D. thesis, Swedish Institute of Space Physics, Kiruna, Sweden, 2008.

Kero, J., Szasz, C., Pellinen-Wannberg, A., Wannberg, G., Westman, A., and Meisel, D. D.: Determination of meteoroid physical properties from tristatic radar observations, Ann. Geophys., 26, 2217-2228, doi:10.5194/angeo-26-2217-2008, 2008.

Kero, J., Szasz, C., Nakamura, T., Meisel, D. D., Ueda, M., Fujiwara, Y., Terasawa, T., Miyamoto, H., and Nishimura, K.: First results from the 2009-2010 MU radar head echo observation programme for sporadic and shower meteors: the Orionids 2009, MNRAS, 416, 2550-2559, doi:10.1111/j.13652966.2011.19146.x, 2011. 
Kero, J., Fujiwara, Y., Abo, M., Szasz, C., and Nakamura, T.: MU radar head echo observations of the 2011 October Draconids, MNRAS, 424, 1799-1806, doi:10.1111/j.13652966.2012.21255.x, 2012a.

Kero, J., Szasz, C., Nakamura, T., Meisel, D. D., Ueda, M., Fujiwara, Y., Terasawa, T., Nishimura, K., and Watanabe, J.: The 2009-2010 MU radar head echo observation programme for sporadic and shower meteors: radiant densities and diurnal rates, MNRAS, 425, 135-146, doi:10.1111/j.13652966.2012.21407.x, 2012b.

Kero, J., Szasz, C., Nakamura, T., Terasawa, T., Miyamoto, H., and Nishimura, K.: A meteor head echo analysis algorithm for the lower VHF band, Ann. Geophys., 30, 639-659, doi:10.5194/angeo-30-639-2012, 2012c.

Kresáková, M.: The Magnitude Distribution of Meteors in Meteor Streams, Contributions of the Astronomical Observatory Skalnate Pleso, 3, 75-112, 1966.

Latteck, R., Singer, W., Rapp, M., and Renkwitz, T.: MAARSY the new MST radar on Andøya/Norway, Adv. Radio Sci., 8, 219 224, doi:10.5194/ars-8-219-2010, 2010.

Licandro, J., Campins, H., Mothé-Diniz, T., Pinilla-Alonso, N., and de León, J.: The nature of comet-asteroid transition object (3200) Phaethon, A\&A, 461, 751-757, doi:10.1051/00046361:20065833, 2007.

Malhotra, A. and Mathews, J. D.: A statistical study of meteoroid fragmentation and differential ablation using the Resolute Bay Incoherent Scatter Radar, J. Geophys. Res. (Space Physics), 116, A04316, doi:10.1029/2010JA016135, 2011.

Mathews, J. D., Meisel, D. D., Hunter, K. P., Getman, V. S., and Zhou, Q.: Very High Resolution Studies of Micrometeors Using the Arecibo $430 \mathrm{MHz}$ Radar, Icarus, 126, 157-169, doi:10.1006/icar.1996.5641, 1997.

Mathews, J. D., Briczinski, S. J., Meisel, D. D., and Heinselman, C. J.: Radio and meteor science outcomes from comparisons of meteor radar observations at AMISR Poker Flat, Sondrestrom and Arecibo, EM\&P, 102, 365-372, doi:10.1007/s11038-0079168-0, 2008.

McIntosh, B. A. and Simek, M.: Geminid meteor stream - Structure from 20 years of radar observations, Bulletin of the Astronomical Institutes of Czechoslovakia, 31, 39-50, 1980.

Nishimura, K., Sato, T., Nakamura, T., and Ueda, M.: High sensitivity radar-optical observations of faint meteors, IEICE Trans. Comm., E84-C, 1877-1884, 2001.

Olsson-Steel, D.: The dispersal of the Geminid meteoroid stream by radiative effects, MNRAS, 226, 1-17, 1987.

Pellinen-Wannberg, A. and Wannberg, G.: Meteor observations with the European Incoherent Scatter UHF radar, J. Geophys. Res., 99, 11379-11390, 1994.

Plavcová, Z.: Radio-echo observations of the Geminid meteor stream in 1959, Bulletin of the Astronomical Institutes of Czechoslovakia, 13, 176, 1962.

Rapp, M. and Robertson, S.: Preface: ECOMA/MASS: Aerosol particles near the polar summer mesopause, Ann. Geophys., 27, 2009.

Rapp, M., Plane, J. M. C., Strelnikov, B., Stober, G., Ernst, S., Hedin, J., Friedrich, M., and Hoppe, U.-P.: In situ observations of meteor smoke particles (MSP) during the Geminids 2010: constraints on MSP size, work function and composition, Ann. Geophys., 30, 1661-1673, doi:10.5194/angeo-30-1661-2012, 2012.
Rendtel, J., Arlt, R., and McBeath, A.: Handbook for Visual Meteor Observers, International Meteor Organization, Potsdam, 1995.

Ryabova, G. O.: Age of the Geminid Meteor Stream (Review), Solar System Research, 33, 224-238, 1999.

Ryabova, G. O.: Mathematical model of the Geminid meteor stream formation, in: Meteoroids 2001 Conference, edited by Warmbein, B., vol. 495 of ESA Special Publication, pp. 77-81, 2001.

Ryabova, G. O.: Mathematical modelling of the Geminid meteoroid stream, MNRAS, 375, 1371-1380, doi:10.1111/j.13652966.2007.11392.x, 2007.

Ryabova, G. O.: On the possible ejection of meteoroids from asteroid (3200) Phaethon in 2009, MNRAS, 423, 2254-2259, doi:10.1111/j.1365-2966.2012.21033.x, 2012.

Sato, T., Nakamura, T., and Nishimura, K.: Orbit Determination of Meteors Using the MU Radar, IEICE TRANSACTIONS on Communications, E83-B, 1990-1995, 2000.

Sekanina, Z.: Statistical Model of Meteor Streams. II. Major Showers, Icarus, 13, 475-493, doi:10.1016/0019-1035(70)90094-1, 1970.

Sekanina, Z.: Statistical model of meteor streams. IV - A study of radio streams from the synoptic year, Icarus, 27, 265-321, doi:10.1016/0019-1035(76)90009-9, 1976.

Simek, M. and McIntosh, B. A.: Geminid meteor stream - Activity as a function of particle size, Bulletin of the Astronomical Institutes of Czechoslovakia, 40, 288-298, 1989.

Stober, G., Jacobi, C., and Singer, W.: Meteoroid mass determination from underdense trails, J. Atmos. Solar-Terr. Phys., 73, 895-900, doi:10.1016/j.jastp.2010.06.009, 2011.

Stober, G., Jacobi, C., Matthias, V., Hoffmann, P., and Gerding, M.: Neutral air density variations during strong planetary wave activity in the mesopause region derived from meteor radar observations, J. Atmos. Solar-Terr. Phys., 74, 55-63, doi:10.1016/j.jastp.2011.10.007, 2012.

Stohl, J. and Porubcan, V.: Orionid Meteor Shower - Activity and Magnitude Description, Contributions of the Astronomical Observatory Skalnate Pleso, 10, 39-51, 1981.

Szasz, C.: Radio meteors above the Arctic Circle: radiants, orbits and estimated magnitudes, Ph.D. thesis, Swedish Institute of Space Physics, Kiruna, Sweden, 2008.

Szasz, C., Kero, J., Meisel, D. D., Pellinen-Wannberg, A., Wannberg, G., and Westman, A.: Orbit characteristics of the tristatic EISCAT UHF meteors, MNRAS, 388, 15-25, doi:10.1111/j.1365-2966.2008.13374.x, 2008.

Uchiyama, S.: Geminids ZHR activity profiles as a function of magnitude, WGN, Journal of the International Meteor Organization, 38, 31-35, 2010.

Verniani, F.: An Analysis of the Physical Parameters of 5759 Faint Radio Meteors, J. Geophys. Res., 78, 8429-8462, doi:10.1029/JB078i035p08429, 1973.

Verniani, F. and Hawkins, G. S.: On the Ionizing Efficiency of Meteors, ApJ, 140, 1590-1600, 1964.

Westman, A., Wannberg, G., and Pellinen-Wannberg, A.: Meteor head echo altitude distributions and the height cutoff effect studied with the EISCAT HPLA UHF and VHF radars, Ann. Geophys., 22, 1575-1584, doi:10.5194/angeo-22-1575-2004, 2004.

Wetherill, G. W.: Sun-Approaching Bodies: Neither Cometary Nor Asteroidal?, Meteoritics, 21, 537, 1986.

Wiegert, P. A., Houde, M., and Peng, R.: An upper limit on gas production from 3200 Phaethon, Icarus, 194, 843-846, 
doi:10.1016/j.icarus.2007.12.013, 2008.

Wyatt, S. P. and Whipple, F. L.: The Poynting-Robertson effect on meteor orbits, ApJ, 111, 134-141, 1950.

Younger, P. T., Astin, I., Sandford, D. J., and Mitchell, N. J.: The sporadic radiant and distribution of meteors in the atmosphere as observed by VHF radar at Arctic, Antarctic and equatorial latitudes, Ann. Geophys., 27, 2831-2841, doi:10.5194/angeo-272831-2009, 2009.
Zvolankova, J.: Dependence of the observed rate of meteors on the Zenith distance of the radiant, B. Astronomical Institutes of Czechoslovakia, 34, 122-128, 1983. 